\title{
Treatment of denture-related stomatitis improves endothelial function assessed by flow-mediated vascular dilation
}

\author{
Grzegorz Osmenda ${ }^{1}$, Joanna Maciąg ${ }^{2}$, Grzegorz Wilk ${ }^{1}$, Anna Maciąg ${ }^{3}$, Daniel Nowakowski², \\ Jolanta Loster ${ }^{4}$, Elżbieta Dembowska ${ }^{5}$, Douglas Robertson ${ }^{6}$, Tomasz Guzik ${ }^{1,7}$,
} Marta Cześnikiewicz-Guzikº

\author{
${ }^{1}$ Department of Internal and Agricultural Medicine, Jagiellonian University \\ Collegium Medicum, Krakow, Poland \\ 2Department of Dental Prophylaxis and Experimental Dentistry, Dental School, \\ Jagiellonian University, Krakow, Poland \\ 3Zbigniew Żak Voivodeship Dental Clinic, Krakow, Poland \\ ${ }^{4}$ Department of Prosthodontics, Dental School, Jagiellonian University, Krakow, Poland \\ ${ }^{5}$ Department of Periodontology, Dental School, Pomeranian Medical University, \\ Szczecin, Poland \\ ${ }^{6}$ Oral Sciences Research Group, Glasgow Dental School, School of Medicine, College \\ of Medical, Veterinary and Life Sciences, University of Glasgow, Glasgow, UK \\ ${ }^{7}$ Institute of Cardiovascular and Medical Sciences, University of Glasgow, Glasgow, UK
}

Submitted: 20 August 2015

Accepted: 1 December 2015

Arch Med Sci 2017; 13, 1: 66-74

DOI: 10.5114 /aoms.2017.64715

Copyright (c) 2016 Termedia \& Banach

\section{Abstract}

Introduction: The presence of oral inflammation has recently been linked with the pathogenesis of cardiovascular diseases. While numerous studies have described links between periodontitis and endothelial dysfunction, little is known about the influence of denture-related stomatitis (DRS) on cardiovascular risk. Therefore, the aim of this study was to determine whether the treatment of DRS can lead to improvement of the clinical measures of vascular dysfunction.

Material and methods: The DRS patients were treated with a local oral antifungal agent for 3 weeks. Blood pressure, flow-mediated dilatation (FMD) and nitroglycerine-mediated vascular dilatation (NMD) were measured during three study visits: before treatment, one day and two months after conclusion of antifungal therapy.

Results: Flow-mediated dilatation measurements showed significant improvement of endothelial function 2 months after treatment (FMD median $5 \%, 95 \mathrm{Cl}: 3-8.3$ vs. $11 \%, 95 \% \mathrm{Cl}: 8.8-14.4 ; p<0.01$ ), while there was no difference in control, endothelium-independent vasorelaxations (NMD; median $=15.3 \%, 95 \% \mathrm{Cl}: 10.8-19.3$ vs. $12.7 \%, 95 \% \mathrm{Cl}: 10.6-15 ; p=0.3)$. Other cardiovascular parameters such as systolic (median $=125 \mathrm{~mm} \mathrm{Hg} ; 95 \% \mathrm{Cl}$ : $116-129$ vs. $120 \mathrm{~mm} \mathrm{Hg}, 95 \% \mathrm{Cl}: 116-126 ; p=0.1$ ) as well as diastolic blood pressure and heart rate (median $=65.5 \mathrm{bpm}, 95 \% \mathrm{Cl}: 56.7-77.7$ vs. $71 \mathrm{bpm}$, $95 \% \mathrm{Cl}: 66.7-75 ; p=0.5$ ) did not change during or after the treatment.

Conclusions: Treatment of DRS is associated with improvement of endothelial function. Since endothelial dysfunction is known to precede the development of severe cardiovascular disorders such as atherosclerosis and hypertension, patients should be more carefully screened for DRS in general dental practice, and immediate DRS treatment should be advised.

Key words: denture, Candida albicans, nystatin, blood pressure, endothelial dysfunction.

\section{Corresponding author:}

Marta Cześnikiewicz-Guzik

Department

of Dental Prophylaxis

and Experimental Dentistry

Dental School

Jagiellonian University

Oral Sciences

Research Group

Glasgow Dental School

School of Medicine

College of Medical,

Veterinary and Life Sciences

University of Glasgow

Phone: +48 666833950

E-mail: marta.czesnikiewiczguzik@uj.edu.pl,

marta.czesnikiewicz-guzik@

glasgow.ac.uk 


\section{Introduction}

Inflammatory processes in the oral cavity have recently been linked to the pathogenesis of systemic diseases, such as cardiovascular diseases [1-5] or rheumatoid arthritis [6]. Most studies have focused on the links between periodontitis and vascular disease including atherosclerosis [1-5]. Periodontal disease is an important risk factor for these disorders, and it has been shown that intensive hygiene phase therapy alleviates endothelial dysfunction in a long-term follow up, with clinical benefit lasting up to 6 months after treatment [7]. Mechanisms which link oral inflammation and cardiovascular effects are not yet fully described, but one of the most important hypotheses is the preactivation of the immune system. Local inflammation in the oral cavity may trigger systemic inflammation, with the production of interleukin (IL)-6, C-reactive protein (CRP), tumour necrosis factor (TNF)- $\alpha$ and other proinflammatory cytokines [7, 8], as well as activation of a cellular immune response [9].

Similarly to periodontitis, denture-related stomatitis (DRS) is an inflammatory process localized in the oral cavity. Denture-related stomatitis is an important clinical issue in elderly patients, making it particularly relevant in the light of the ageing of western societies. Denture-related stomatitis affects up to $70 \%$ of patients during the course of life [10, 11], with erythema and swelling of palatal mucosa being the main clinical symptoms. Because of the non-specificity of these symptoms, this disease often remains undiagnosed and untreated for long periods of time. The etiology of DRS is multifactorial [10] but is associated with long-term and continuous use of dentures and poor denture and oral hygiene habits which promote disease development $[10,12]$. The presence of denture, together with other favourable conditions, such as low salivary $\mathrm{pH}$, regular sugar consumption [13] and local immune surveillance changes [14], promote excessive growth of Candida albicans, which is a fungal component of the physiological microflora of the human oral cavity $[15,16]$. Consequently, Candida infection develops and DRS occurs.

While numerous studies have focused on the links between periodontitis and endothelial dysfunction, little is known about the links between other forms of oral infection and inflammation in the context of cardiovascular risk. Interestingly, increased cardiovascular risk has been shown also for caries $[17,18]$, as well as endodontic infection [19-21]. These diseases are all caused by bacterial infections, while fungal oral cavity infections have not been studied in this context. We found only one study, by Buhlin et al. [2], describing a positive association between the use of dentures and the prevalence of cardiovascular diseases. However, the group defined as "denture users" was very heterogeneous, and it remains unclear how many denture users suffered from DRS, as the study was based on a patient questionnaire, making it difficult to draw firm conclusions. More recently we have described the presence of vascular endothelial dysfunction in DRS patients, compared to the denture patients with healthy oral mucosa [22]. Since endothelial dysfunction is known to precede the development of hypertension and atherosclerosis [23] with its complications, such as heart attack and stroke, it is essential to clarify the influence of DRS on the endothelial function. As the prevalence of DRS in elderly denture users is high, this could make DRS a very significant novel cardiovascular risk factor.

To study the cause and effect relationship between DRS and endothelial dysfunction, an interventional study is necessary. Therefore, the aim of this study was to determine whether the treatment of local inflammation associated with DRS influenced the clinical measures of vascular dysfunction, such as endothelial function or blood pressure.

\section{Material and methods}

\section{Patients and clinical evaluation}

One hundred and twenty-eight patients using dental prostheses for at least 6 months were screened for clinical symptoms of oral mucosal inflammation such as erythema, swelling, discomfort and pain in the palatal area. In patients with teeth present clinical periodontal pocket depth was measured at six points around each tooth to exclude the presence of periodontitis. Patients with one or more periodontal pocket depth $\geq 4 \mathrm{~mm}$ were excluded from the studies. Exclusion criteria also included acute inflammatory disorders other than DRS, neoplastic disease or its relapses and chemotherapy courses less than 5 years before the enrolment, antibiotics regimen in the preceding 4 weeks or anti-inflammatory drugs (steroids and non-steroidal, excluding aspirin in doses lower than $80 \mathrm{mg}$ ) in the preceding 2 months before the enrolment. Patients with a history of myocardial infarction, acute coronary incident or vascular inflammation in 5 weeks or less before the enrolment, chronic haematological disorders and immunodeficiency or major medication changes within 5 weeks before or during the study were also excluded.

Swabs from the inflamed area were obtained from $n=20$ subjects for the microbiological culture for the presence of Candida species to confirm the clinical diagnosis of DRS. Seventeen patients were recruited to the interventional study when clinical diagnosis was confirmed by the culture of Candida species from the palatal mu- 


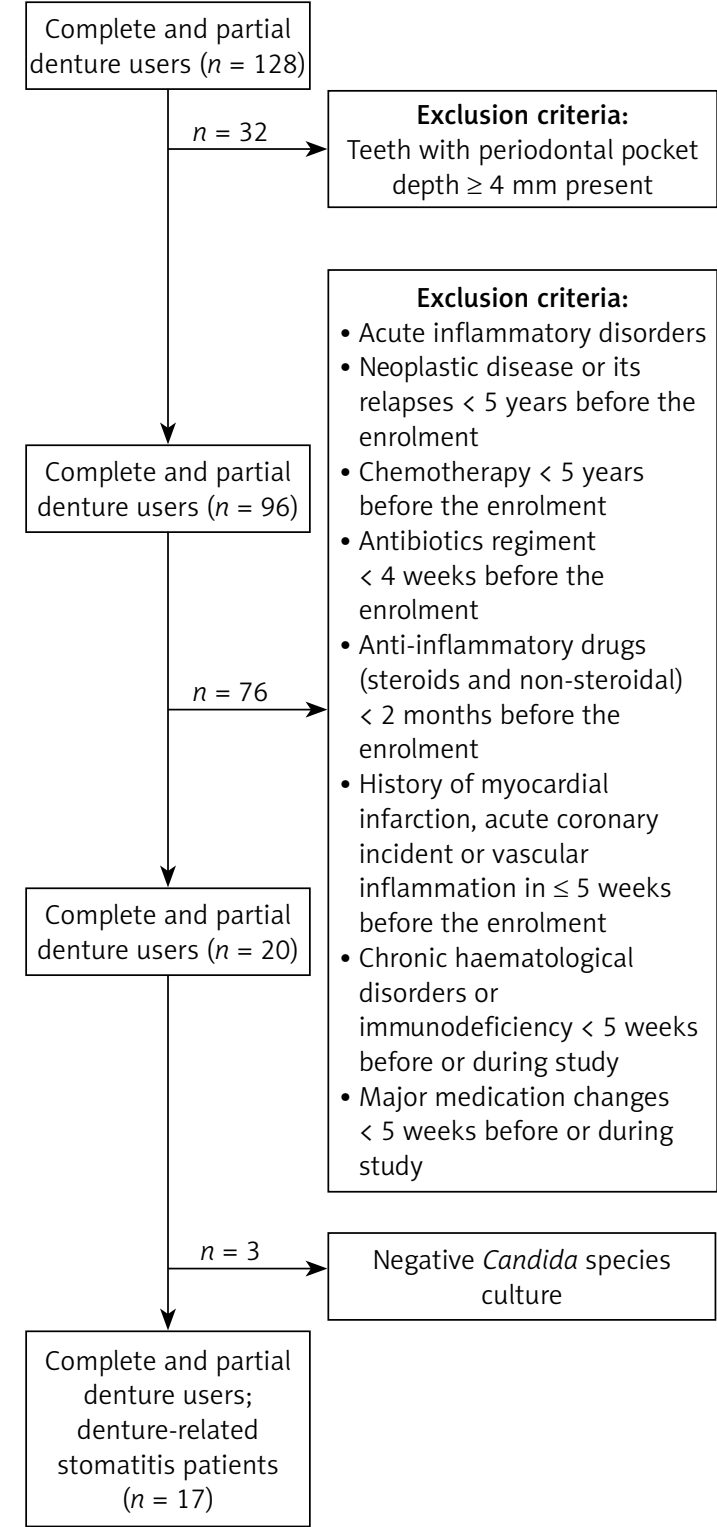

Figure 1. Study design for screening and recruitment of the DRS patients

cosa (Figure 1). Treatment of oral fungal infection included application of nystatin (100 $000 \mathrm{lU} /$ $\mathrm{ml}$ ) every $6 \mathrm{~h}$ for 2 weeks on the infected area of the mucous membrane of the palate. This was done by administrating 5 drops of nystatin suspension on the palatal side of the denture and placing it on the palatal mucosa. Patients' dentures were evaluated by a clinician, and if indicated, dentures were replaced for the new ones during nystatin treatment. Use of any other concomitant medications was monitored and remained unchanged throughout the study period. Blood pressure, flow-mediated (FMD) and nitroglycerine-mediated (NMD) vascular dilatation data were measured in all patients during three visits: the first data set was obtained before commencement of the treatment regimen (visit 1), the second immediately after conclusion of treatment to assess the direct influence of therapy (visit 2), and the third 2 months after completion of treatment to assess longterm effects of termination of infection (visit 3). At the same time, a swab from mucosal tissue was taken during visit 2 to confirm the effectiveness of the antimicrobial treatment and Candida species eradication. While randomization to a placebo group would be valuable, it has been deemed unethical in patients clearly requiring treatment.

The study was reviewed and approved by the local ethics committee of Jagiellonian University. Written informed consent was obtained from all patients, and the study was conducted in accordance with the Declaration of Helsinki. This study has been registered in ClinicalTrials.gov (identification no. NCT02166450).

\section{Microbiological investigations}

Swabs were taken from the hard palate (between the second and third palatal fold). Samples were collected after an overnight fast and after at least $6 \mathrm{~h}$ of denture using, without its cleaning, use of adhesives or rinsing the mouth with disinfectants. The material was collected in accordance with the general principles of microbial material collection. Candida identification was made according to the colour of the colony on CHROMagar Candida medium (Graso Biotech, Starogard Gdanski, Poland) after 36 to 48 h of incubation at $36^{\circ} \mathrm{C}$.

\section{Clinical data}

Patients' blood pressure (systolic, diastolic) was monitored for $24 \mathrm{~h}$ using an ambulatory blood pressure monitoring system (Spacelabs90217, Ultralite). Systolic, diastolic and mean arterial pressures were recorded every 20 min for $24 \mathrm{~h}$. Day and night averages were calculated. One patient did not agree to wear the ambulatory blood pressure monitoring system. Major risk factors for both atherosclerosis and DRS were recorded based on patient medical records and detailed patient medical history obtained during the interview. Clinical risk factors were defined as follows: hyperlipidaemia (total plasma cholesterol level $>5 \mathrm{mmol} / \mathrm{l}$ and/or triglycerides level $>1.7 \mathrm{mmol} / \mathrm{l}$ ); diabetes (fasting glucose level $\geq 7 \mathrm{mmol} / \mathrm{l}$ or glycated haemoglobin $\left(\mathrm{HbA}_{1 \mathrm{c}}\right)>6.5 \%$ or current treatment with insulin or oral hypoglycaemic agents); hypertension (blood pressure $\geq 140$ / $90 \mathrm{~mm} \mathrm{Hg}$ or current treatment with antihypertensive agents), and smoking (current or within last 6 months) [24]. Blood samples were obtained from the antecubital vein and lipoprotein profile was assessed by measurements of triglycerides, 
total cholesterol, and low- and high-density lipoprotein cholesterol fractions. C-reactive protein concentration was also assessed.

\section{Subclinical atherosclerosis}

To characterize subclinical atherosclerosis, assessment of carotid artery intima-media thickness was performed. It was measured at 12 different points ( $2 \mathrm{~cm}$ below common carotid arteries bulbs, ca. every $1 \mathrm{~cm}$, omitting visible coronary plaques), on right and left common carotid artery, measuring the distance between the border between the artery lumen and carotid artery intima and second bright line-m (border between media and adventitia) as described previously [25].

\section{Endothelial function measurement}

The FMD method was used to determine the vascular endothelial function and NMD for measuring endothelial-independent vasodilatation. Measurements were conducted using the Toshiba Xario Diagnostic Ultrasound System after 1, 2 and 4-5 min after manometer cuff deflation or sublingual administration of nitroglycerine and presented as percentage of the diameter of the artery before intervention. Method validation in our laboratory has been described before [25]. Observers were blinded regarding oral status of the patients.

\section{Statistical analysis}

Assessment of normality of distribution for all tested continuous variables was conducted by the Shapiro-Wilk test. Variables whose distributions were not consistent with normal distribution are presented as the median and $25^{\text {th }}(\mathrm{Q} 1)$ and $75^{\text {th }}$ (Q3) percentiles, and values for subsequent visits were compared by the Friedman test, with posthoc analysis in case of detecting significance. Variables with normal distribution are presented as mean \pm standard deviation (SD). All variables with normal distributions described baseline group characteristics, and comparison between visits was not applicable. The method of presentation of results is given for each variable in the text. Values of $p<0.05$ were considered statistically significant. All analyses were performed with StatSoft Statistica software (StatSoft, Inc., Tulsa, OK, USA).

\section{Results}

\section{Study population characteristics}

Clinical characteristics of studied patients are shown in Table I. Patients were mostly women $(n=14 ; 82.3 \%)$ with mean age $62.8 \pm 6.0$ years and mean BMI $28.9 \pm 5.8 \mathrm{~kg} / \mathrm{m}^{2} .35 .3 \%$ of patients were current smokers and diabetics, which is of particular significance, as these features are key risk fac-
Table I. Clinical characteristics of studied groups

\begin{tabular}{|c|c|}
\hline Parameter & Result \\
\hline Number of patients & 17 \\
\hline Age [years] & $62.8(6.0)$ \\
\hline Gender $(\mathrm{M}: \mathrm{F})$ & $3: 14$ \\
\hline \multicolumn{2}{|l|}{ Maxillary dentures: } \\
\hline Partial & $3(18 \%)$ \\
\hline Complete & $14(82 \%)$ \\
\hline \multicolumn{2}{|l|}{ Mandibular dentures: } \\
\hline Partial & $3(18 \%)$ \\
\hline Complete & $14(82 \%)$ \\
\hline BMI: & $28.9(5.8)$ \\
\hline Overweight & $5(29.4 \%)$ \\
\hline Obesity & $6(35.3 \%)$ \\
\hline \multicolumn{2}{|l|}{ Cigarette smoking: } \\
\hline Current smokers & $6(35.3 \%)$ \\
\hline Past smokers & $1(5.9 \%)$ \\
\hline \multicolumn{2}{|l|}{ Accompanying diseases: } \\
\hline Diabetes & $6(35.3 \%)$ \\
\hline Coronary artery disease & $7(41.2 \%)$ \\
\hline Hypertension: & $15(88.2 \%)$ \\
\hline Controlled & $7(41.2 \%)$ \\
\hline Untreated & $8(47.1 \%)$ \\
\hline Hyperlipidaemia & $12(70.6 \%)$ \\
\hline \multicolumn{2}{|l|}{ Medications: } \\
\hline ACE inhibitor & $9(52.9 \%)$ \\
\hline Acetylsalicylic acid & $8(47.1 \%)$ \\
\hline$\alpha$-Blocker & $1(5.9 \%)$ \\
\hline$\beta$-Blocker & $8(47.1 \%)$ \\
\hline Calcium antagonist & $6(35.3 \%)$ \\
\hline Diuretic & $9(52.9 \%)$ \\
\hline Statin & $11(64.7 \%)$ \\
\hline Insulin & $1(5.9 \%)$ \\
\hline Oral antidiabetic agents & $4(23.5 \%)$ \\
\hline $\begin{array}{l}\text { Carotid artery intima media thickness } \\
{[\mathrm{mm}]}\end{array}$ & $0.85(0.22)$ \\
\hline Atherosclerotic plaque presence & $7(41.2 \%)$ \\
\hline \multicolumn{2}{|l|}{ Blood lipid characteristics: } \\
\hline Total cholesterol [mmol/l] & $5.7(1.3)$ \\
\hline Triglycerides [mmol/l] & $1.7(0.7)$ \\
\hline HDL cholesterol [mmol/l] & $1.4(0.4)$ \\
\hline LDL cholesterol [mmol/l] & $3.5(1.1)$ \\
\hline Blood CRP levels $[\mathrm{mg} / \mathrm{ml}]$ & $2.5(3.4)$ \\
\hline
\end{tabular}

Data are presented as mean (SD) or $n$ (\%). ACE - angiotensin converting enzyme, $B M I$ - body mass index, CRP - C-reactive protein, $F$ - females, $H D L$ - high-density lipoproteins, $L D L-$ lowdensity lipoproteins, $M$ - males. 
tors for DRS. Eighty-eight percent of patients were hypertensive. Only 3 out of 17 patients did not use any medications, and the most commonly used were statins ( $65 \%$ of patients), followed by angiotensin-converting enzyme (ACE) inhibitors and diuretics (53\% each) and $\beta$-blockers and acetylsalicylic acid (47\%). Use of cardiovascular medications was monitored and remained unchanged throughout the study. Despite the fact that almost half of the recruited patients (41\%) presented with atherosclerotic lesions, mean carotid artery intima-media thickness did not exceed standard values.

\section{Clinical effectiveness of treatment}

Treatment was effective in all studied patients and improvement was seen in both the clinical signs and negative oral Candida swabs. This improvement persisted during subsequent twomonth observation, with no recurrences of DRS (Table II).

\section{Vascular function assessment}

Flow-mediated dilatation measurements, which indicate endothelium-dependent vessel function and its ability to constrict and dilate depending on the physiological needs of the cardiovascular system, showed significant improvement in median percentage of arterial dilation in response to flow in the DRS patients between the visit before and 2 months after treatment (Figure 2 A). Furthermore, after completion of the treatment the endothelium-dependent vascular function continued to improve, showing that resolution of chronic inflammation in the oral cavity brings significant benefits for the endothelial physiology (Figure $2 \mathrm{~A}$ ). At the same time, there was no difference in values of endothelium-independent vasodilatation (NMD) (Figure 2 B).

\section{Blood pressure analysis}

Median systolic and diastolic blood pressure of DRS patients showed no significant changes throughout treatment (Figures $3 \mathrm{~A}, \mathrm{C}$ ). Similarly, no differences were observed in the median mean arterial and pulse pressure (Figures 3 B, D). Treatment of DRS did not affect systolic and diastolic blood pressure values during either activity or rest periods (daytime/night-time blood pressure; Figure 3).

Table II. Effectiveness of DRS treatment

\begin{tabular}{|c|c|c|c|}
\hline Variable & $\begin{array}{l}\text { Pre-treatment } \\
\text { screening }\end{array}$ & $\begin{array}{l}\text { One day after therapy } \\
\text { completion }\end{array}$ & $\begin{array}{l}\text { Two months after therapy } \\
\text { completion }\end{array}$ \\
\hline Redness of the mucosa & $100 \%$ & $0 \%$ & $0 \%$ \\
\hline Swelling of the mucosa & $47 \%$ & $6 \%$ & $0 \%$ \\
\hline Discomfort and pain & $76 \%$ & $18 \%$ & $6 \%$ \\
\hline Positive culture of Candida species & $100 \%$ & $0 \%$ & $\mathrm{~N} / \mathrm{A}$ \\
\hline
\end{tabular}

Data are presented as \% of total number of patients. N/A - not applicable, microbiological investigations was not performed.

A

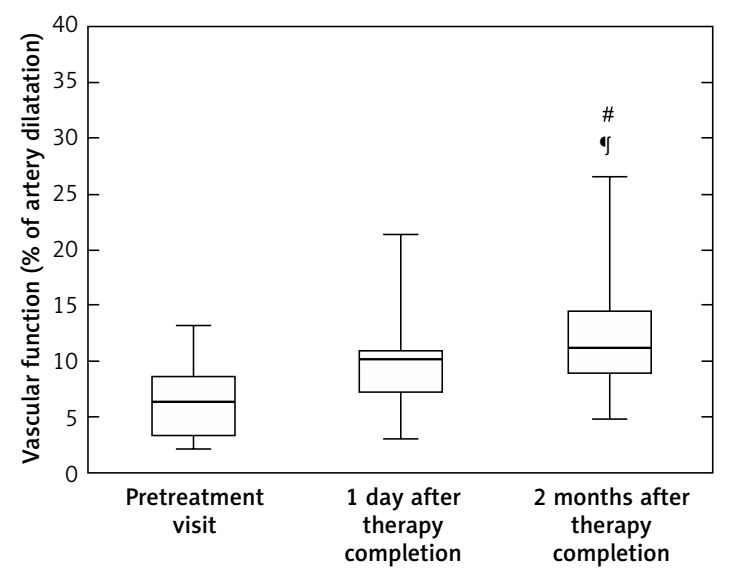

B

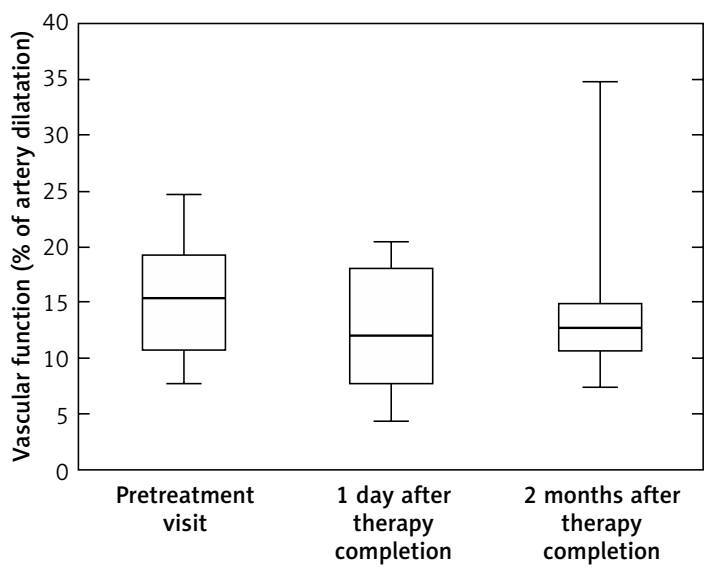

Figure 2. Vascular dysfunction in DRS patients treated with nystatin. Vascular endothelium-dependent flow-mediated dilatation (A) and endothelium-independent nitroglycerine-mediated dilatation (B) parameters were assessed by ultrasonography before starting nystatin therapy, immediately after finishing it and 2 months after therapy completion

Results presented as median (box: Q1-Q3; whisker: minimal and maximal measurements). ${ }^{*} p<0.05$ for comparison of $2^{\text {nd }}$ and $3^{\text {rd }}$ visit; " $p<0.05$ for comparison of $1^{\text {st }}$ and $3^{\text {rd }}$ visit. 
A

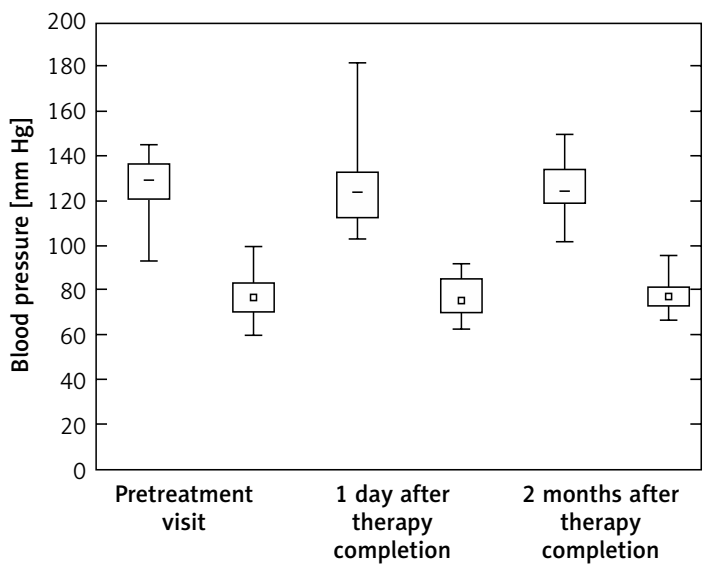

C

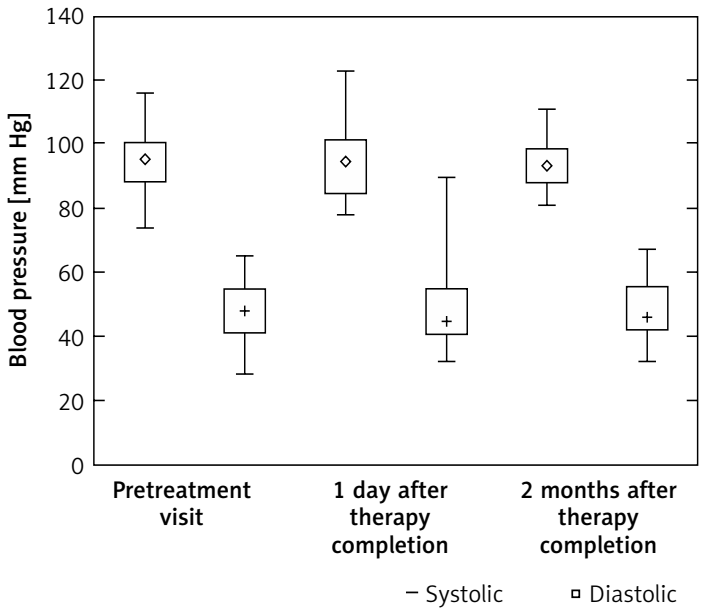

B

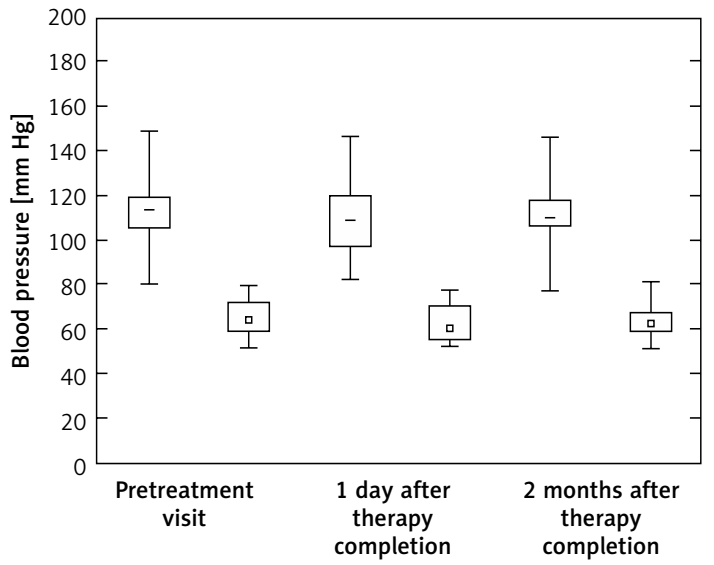

D

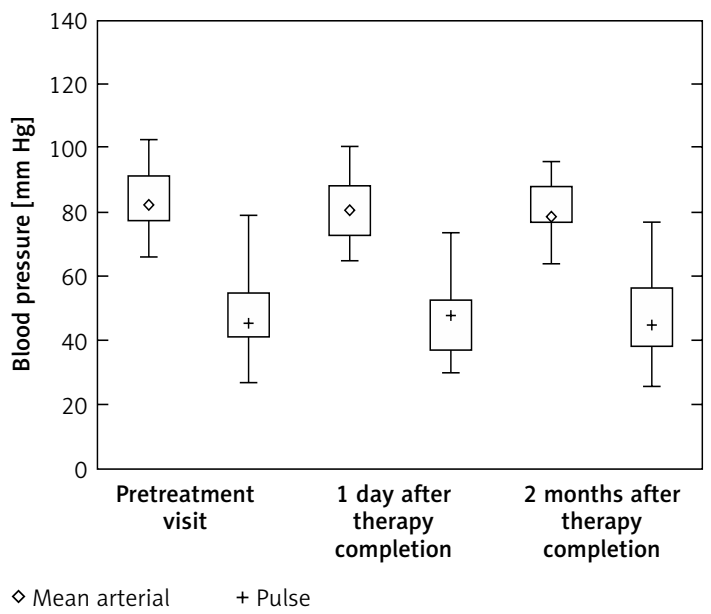

Figure 3. Blood pressure characteristics in periods of rest and activity in DRS patients treated with nystatin. Comparison of systolic and diastolic blood pressure $(\mathbf{A}, \mathbf{C})$ and mean arterial and pulse pressure (B, D) in DRS patients before starting nystatin therapy, immediately after finishing it and 2 months after therapy completion during activity and rest periods of the day. 24-hour measurement of blood pressure was performed using an ambulatory blood pressure monitoring system

Results presented as median (box: Q1-Q3; whisker: minimal and maximal measurements).

\section{Discussion}

The links between the general and oral health have become the object of keen interest among researchers and clinicians in recent years. The impact of oral conditions on various diseases, including rheumatoid arthritis [26], obesity [27], peptic ulcers [28], negative pregnancy outcomes [29], diabetes [30] and in epilepsy [31] has been investigated. However, most studies have focused predominantly on bacterial infections in the oral cavity such as periodontitis, caries or endodontic infections. At the same time, DRS, being inflammation of oral mucosa related primarily to fungal infection, has not been widely studied in relation to vascular disease to date.

In our study, we demonstrated for the first time that the benefits from successful treatment of DRS extend beyond the oral cavity and lead to an improvement in vascular dysfunction. Endothelium-dependent, flow-induced brachial artery dilatation steadily improved during the treatment and reached statistical significance 2 months after finishing the therapy, showing a significant improvement in vascular function, while the control, nitroglycerine-induced endothelium-independent vasodilatation remained unchanged. This observation is therapeutically important and extends our recent report that vascular function is impaired in DRS [22]. While the previously described link could have been attributed to confounding factors, such as diabetes, the results presented in this paper show that endothelial function in DRS is directly related to oral infection and removal of this pathology results in significant improvement of vascular health.

While it was not possible to study a placebo group for ethical reasons, this effect is unlikely to 
be related to the placebo effect or direct nystatin use, as the improvement of endothelial function was maintained for subsequent months after oral inflammation was removed.

While our study is the first to show the effects of DRS therapy on endothelial function, previous studies have shown similar improvements in relation to other oral diseases, particularly periodontitis. Two research groups have presented results showing that subjects with advanced periodontal disease exhibit worse endothelial function when compared to the healthy controls [32]. Moreover, periodontal treatment leads to the improvement of endothelial function as a long-term outcome [7, 33]. One of these studies was a randomized controlled trial which showed that such improvement provides clinical benefit for up to 6 months after intensive treatment of chronic periodontitis [7].

The importance of our observation is particularly significant because it its known that severity of endothelial dysfunction not only correlates with the development of coronary artery disease, but may also predict future cardiovascular events. This is also of great importance given that DRS is one of the most common oral disorders, affecting $40-70 \%$ of elderly denture wearers $[10,11]$, and its frequency might possibly increase because of ageing of the world population. In the light of these facts, the results of our study have clinically important implications for the management of patients with DRS. Patients with DRS should be identified early through screening during patients' visits to their dentist and where diagnosed should be treated timely and thoroughly in order to eliminate this as a source of systemic inflammation.

In our study, we did not observe a significant influence of DRS treatment on blood pressure values. Since endothelial dysfunction is known to precede the development of hypertension and atherosclerosis [23], it is possible that the observation period was too short for such effects to become detectable. On the other hand, it is in line with our previous study, where we also did not observe an association between DRS presence and blood pressure values. This aspect was also studied by Buhlin et al. [2], who reported a positive association between using dentures and all cardiovascular diseases, but not for its components, which include elevated blood pressure, myocardial infarction or stroke. These authors combined partially edentulous denture wearers and edentulous patients without dentures, which made this group highly heterogeneous and with potential bias of the presence of periodontitis, which complicated drawing firm conclusions. This contrasts with periodontitis, where links with blood pressure were frequently reported [34]. This may reflect potential differences between these two oral conditions.
One potential mechanism explaining the exacerbation of cardiovascular disorders by inflammatory oral diseases as DRS is immune system activation. However, proving a cause-and-effect relationship between inflammatory diseases of the oral cavity, such as DRS, and cardiovascular health is particularly difficult due to common risk factors including diabetes, hyperlipidaemia, smoking and age [10, 12, 35]. However, the design of our study allowed us to minimize the effect of risk factors, since the only changing variable was oral inflammation. The DRS presence seems to be related to systemic immune response by influencing cytokine production by peripheral immune cells, as shown in in vitro studies, where production of IL-2 by mononuclear blood cells of DRS patients was stimulated in response to Candida albicans [36] and high levels of IL- 6 and TNF- $\alpha$ were observed in the serum of DRS patients [37]. However, systemic activation of the immune response in DRS appears to be much more subtle than that observed in chronic periodontitis, in which levels of IL-6, CRP, TNF- $\alpha$ and other cytokines are elevated $[7,8]$. Likewise, a possible role of reactive oxygen species and enzymes associated with their production, which is important in vascular pathology [38], as well as in other diseases [39], cannot be excluded.

The present study is limited by the fact that we were unable to study a placebo-treated group. It was deemed unethical to leave patients with clearly evident fungal infection without sufficient anti-fungal treatment for the duration of the study. Therefore we had to focus on comparisons in relation to pre-treatment status. While the number of subjects is not very high and the length of follow-up period is not very long, they were sufficient to record a clear, statistically significant improvement of endothelial function, which was the main endpoint of the study. The evidence of continued improvement in endothelial function following the completion of nystatin therapy is of particular importance, because it indicates that the improvement was related to the lack of oral inflammation rather than to a direct effect of nystatin. We monitored the most reliable indicator of endothelial function - flow-mediated dilatation [40]. In future it would be valuable to address some other biomarkers of endothelial health with all the limitations of these, including soluble adhesion molecules, ADMA and von Willebrand factor, as well as others [9, 41-44]. Predominance of women in the study group could also be a limitation to the generalizability of the study findings; however, the study population is consistent with the epidemiology of DRS. This fact should be taken into account before translation of the results to the general population. Moreover, 
the population of elderly women is prone to specific, menopause-related processes, such as osteoporosis [45]. However, given the fact of lack of available data on this aspect and the importance of detected associations, we believe that these findings may become a starting point for further studies, which broaden the knowledge and raise awareness about the associations between DRS and oral health in general and patients' systemic health and well-being.

In conclusion, the most significant observation of our research is improvement of endothelial function in relation to DRS treatment. Since endothelial dysfunction is known to precede the development of more severe cardiovascular disorders, patients should be more carefully screened for DRS in general dental practice, and immediate DRS treatment should be advised in future dental guidelines.

\section{Acknowledgments}

Grzegorz Osmenda and Joanna Maciąg contributed equally to the study.

This study was supported by Public Funds for Science of Republic of Poland grant Nr 2997/B/ P01/2009/36 and the Foundation for Polish Science Welcome Grant (FNP/Welcome/02) (TJG, GO).

\section{Conflict of interest}

The authors declare no conflict of interest.

\section{References}

1. Arbes SJ Jr, Slade GD, Beck JD. Association between extent of periodontal attachment loss and self-reported history of heart attack: an analysis of NHANES III data. J Dent Res 1999; 78: 1777-82.

2. Buhlin K, Gustafsson A, Håkansson J, Klinge B. Oral health and cardiovascular disease in Sweden. J Clin Periodontol 2002; 29: 254-9.

3. Buhlin K, Gustafsson A, Ahnve S, Janszky I, Tabrizi F, Klinge B. Oral health in women with coronary heart disease. J Periodontol 2005; 76: 544-50.

4. Desvarieux M, Demmer RT, Rundek T, et al. Relationship between periodontal disease, tooth loss, and carotid artery plaque: the Oral Infections and Vascular Disease Epidemiology Study (INVEST). Stroke J Cereb Circ 2003; 34: 2120-5.

5. Hung HC, Willett W, Merchant A, Rosner BA, Ascherio A, Joshipura KJ. Oral health and peripheral arterial disease. Circulation 2003; 107: 1152-7.

6. Kobayashi T, Yoshie $\mathrm{H}$. Host responses in the link between periodontitis and rheumatoid arthritis. Curr Oral Health Rep 2015; 2: 1-8.

7. Tonetti MS, D'Aiuto F, Nibali L, et al. Treatment of periodontitis and endothelial function. N Engl J Med 2007; 356: 911-20.

8. Noh MK, Jung M, Kim SH, et al. Assessment of IL-6, IL-8 and TNF-alpha levels in the gingival tissue of patients with periodontitis. Exp Ther Med 2013; 6: 847-51.

9. Chalubinski M, Wojdan K, Dorantowicz R, Jackowska P, Gorzelak P, Broncel M. Comprehensive insight into im- mune regulatory mechanisms and vascular wall determinants of atherogenesis - emerging perspectives of immunomodulation. Arch Med Sci 2013; 9: 159-65.

10. Gendreau L, Loewy ZG. Epidemiology and etiology of denture stomatitis. J Prosthodont Off J Am Coll Prosthodont 2011; 20: 251-60.

11. Kossioni AE. The prevalence of denture stomatitis and its predisposing conditions in an older Greek population. Gerodontology 2011; 28: 85-90.

12. Salerno C, Pascale M, Contaldo M, et al. Candida-associated denture stomatitis. Med Oral Patol Oral Cir Bucal 2011; 16: e139-43.

13. Martori E, Ayuso-Montero R, Martinez-Gomis J, Viñas M, Peraire M. Risk factors for denture-related oral mucosal lesions in a geriatric population. J Prosthet Dent 2014; 111: 273-9.

14. Gasparoto $T H$, Sipert CR, de Oliveira CE, et al. Salivary immunity in elderly individuals presented with Candida-related denture stomatitis. Gerodontology 2012; 29: e331-9.

15. Campos MS, Marchini L, Bernardes LAS, Paulino LC, Nobrega FG. Biofilm microbial communities of denture stomatitis. Oral Microbiol Immunol 2008; 23: 419-24.

16. Ghannoum MA, Jurevic RJ, Mukherjee PK, et al. Characterization of the oral fungal microbiome (mycobiome) in healthy individuals. PLoS Pathog 2010; 6: e1000713.

17. Larsson B, Johansson I, Weinehall L, Hallmans G, Ericson T. Cardiovascular disease risk factors and dental caries in adolescents: effect of a preventive program in Northern Sweden (the Norsjö project). Acta Paediatr 1997; 86: 63-71.

18. Kelishadi R, Mortazavi S, Hossein TR, Poursafa P. Association of cardiometabolic risk factors and dental caries in a population-based sample of youths. Diabetol Metab Syndr 2010; 7: 22.

19. Joshipura KJ, Pitiphat W, Hung HC, Willett WC, Colditz GA, Douglass CW. Pulpal inflammation and incidence of coronary heart disease. J Endod 2006; 32: 99-103.

20. Caplan DJ, Chasen JB, Krall EA, et al. Lesions of endodontic origin and risk of coronary heart disease. J Dent Res 2006; 85: 996-1000.

21. Caplan DJ, Pankow JS, Cai J, Offenbacher S, Beck JD. The relationship between self-reported history of endodontic therapy and coronary heart disease in the Atherosclerosis Risk in Communities Study. J Am Dent Assoc 2009; 140: 1004-12.

22. Maciąg J, Osmenda G, Nowakowski D, et al. Denture-related stomatitis is associated with endothelial dysfunction. BioMed Res Int 2014; 2014: 474016.

23. Guzik TJ, West NE, Black E, et al. Vascular superoxide production by $\mathrm{NAD}(\mathrm{P}) \mathrm{H}$ oxidase: association with endothelial dysfunction and clinical risk factors. Circ Res 2000; 86: E85-90.

24. Perk J, De Backer G, Gohlke H, et al. European Guidelines on cardiovascular disease prevention in clinical practice (version 2012): The Fifth Joint Task Force of the European Society of Cardiology and Other Societies on Cardiovascular Disease Prevention in Clinical Practice (constituted by representatives of nine societies and by invited experts). Atherosclerosis 2012; 223: 1-68.

25. Wilk G, Osmenda G, Matusik P, et al. Endothelial function assessment in atherosclerosis: comparison of brachial artery flow-mediated vasodilation and peripheral arterial tonometry. Pol Arch Med Wewn 2013; 123: 443-52.

26. Bingham CO 3rd, Moni M. Periodontal disease and rheumatoid arthritis: the evidence accumulates for complex pathobiologic interactions. Curr Opin Rheumatol 2013; 25: 345-53. 
27. Zeigler CC, Persson GR, Wondimu B, Marcus C, Sobko T, Modéer T. Microbiota in the oral subgingival biofilm is associated with obesity in adolescence. Obesity 2012; 20: 157-64.

28. Pawlik T, Konturek PC, Konturek JW, et al. Impact of Helicobacter pylori and nonsteroidal anti-inflammatory drugs on gastric ulcerogenesis in experimental animals and in humans. Eur J Pharmacol 2002; 449: 1-15.

29. Kim AJ, Lo AJ, Pullin DA, Thornton-Johnson DS, Karimbux NY. Scaling and root planing treatment for periodontitis to reduce preterm birth and low birth weight: a systematic review and meta-analysis of randomized controlled trials. J Periodontol 2012; 83: 1508-19.

30. Chee B, Park B, Bartold PM. Periodontitis and type II diabetes: a two-way relationship. Int J Evid Based Healthc 2013; 11: 317-29.

31. Costa ALF, Yasuda CL, Shibasaki W, et al. The association between periodontal disease and seizure severity in refractory epilepsy patients. Seizure J Br Epilepsy Assoc 2014; 23: 227-30.

32. Amar S, Gokce N, Morgan S, Loukideli M, Van Dyke TE, Vita JA. Periodontal disease is associated with brachial artery endothelial dysfunction and systemic inflammation. Arterioscler Thromb Vasc Biol 2003; 23: 1245-9.

33. Elter JR, Hinderliter AL, Offenbacher S, et al. The effects of periodontal therapy on vascular endothelial function: a pilot trial. Am Heart J 2006; 151: 47.

34. Leong XF, Ng CY, Badiah B, Das S. Association between hypertension and periodontitis: possible mechanisms. Sci World J 2014; 2014: 768237.

35. Dorocka-Bobkowska B, Zozulinska-Ziolkiewicz D, WieruszWysocka B, Hedzelek W, Szumala-Kakol A, Budtz-Jörgensen $E$. Candida-associated denture stomatitis in type 2 diabetes mellitus. Diabetes Res Clin Pract 2010; 90: 81-6.

36. Rodriguez-Archilla A, Urquia M, Cutando A, Asencio R. Denture stomatitis: quantification of interleukin-2 production by mononuclear blood cells cultured with Candida albicans. J Prosthet Dent 1996; 75: 426-31.

37. Pietruski JK, Pietruska MD, Jabłońska E, Sacha P, Zaremba $M$, Stokowska W. Interleukin 6, tumor necrosis factor alpha and their soluble receptors in the blood serum of patients with denture stomatitis and fungal infection. Arch Immunol Ther Exp (Warsz) 2000; 48: 101-5.

38. Konior A, Schramm A, Czesnikiewicz-Guzik M, Guzik TJ. NADPH oxidases in vascular pathology. Antioxid Redox Signal 2014; 20: 2794-814.

39. Gongora MC, Lob HE, Landmesser U, et al. Loss of extracellular superoxide dismutase leads to acute lung damage in the presence of ambient air: a potential mechanism underlying adult respiratory distress syndrome. Am J Pathol 2008; 173: 915-26.

40. Ertek S, Akgül E, Cicero AF, et al. 25-Hydroxy vitamin D levels and endothelial vasodilator function in normotensive women. Arch Med Sci 2012; 8: 47-52.

41. Głogowska-Ligus J, Dą̧ek J, Zych-Twardowska E, Tkacz M. Expression analysis of intercellular adhesion molecule-2 (ICAM-2) in the context of classical cardiovascular risk factors in acute coronary syndrome patients. Arch Med Sci 2013; 9: 1035-9.

42. Koc-Zorawska E, Malyszko J, Zbroch E, Malyszko J, Mysliwiec M. Vascular adhesion protein-1 and renalase in regard to diabetes in hemodialysis patients. Arch Med Sci 2012; 8: 1048-52.

43. Khazaei M, Nematbakhsh M. Effect of experimentally induced metabolic acidosis on aortic endothelial permeability and serum nitric oxide concentration in normal and high-cholesterol fed rabbits. Arch Med Sci 2012; 8: 719-23.

44. Kokot M, Biolik G, Ziaja D, et al. Endothelium injury and inflammatory state during abdominal aortic aneurysm surgery: scrutinizing the very early and minute injurious effects using endothelial markers - a pilot study. Arch Med Sci 2013; 9: 479-86.

45. Senel K, Baykal T, Seferoglu B, et al. Circulating vascular endothelial growth factor concentrations in patients with postmenopausal osteoporosis. Arch Med Sci 2013; 9: 709-12. 